\title{
Protein analysis for comparison between Salmonellae isolated from different poultry species
}

\author{
Seham A. El-Zeedy ${ }^{1}$, Hussein K. Eldeen ${ }^{1 *}$, Jihan M. Badr ${ }^{2}$ \\ ${ }^{1}$ Veterinary Serum and Vaccine Research Institute, Abbasia, Cairo Egypt. \\ ${ }^{2}$ Animal Health Research Institute, Dokki Giza, Egypt.
}

\begin{abstract}
A total of 620 egg samples from different species (chickens, ducks and ostriches) and 1615 poultry samples (chickens, ducks, pigeons, quails, turkeys and ostriches) were examined for salmonella infection. 12 salmonella isolates were obtained from the egg samples $(1.9 \%)$ and 67 isolates from poultry samples $(\mathbf{4 . 1 \% )}$ ). Salmonella isolates were serotyped into $S$. enteritidis (25 isolates), S. typhimurium (17 isolates), $S$. infantis (12 isolates), $S$. montivideo (7 isolates), 3 isolates for each of $S$. rubislaw and $S$. cerro, 2 isolates for each of $S$. virginia, $S$. agona, $S$. poona, and $S$. derby and 1 isolate for each of $S$. sandiago and $S$. kentucky. The incidence of isolation from different poultry species was discussed in details. Antibiogram of the isolated salmonellae against 10 different antibiotics revealed that norofloxacin, ciprofloxacin, cepheridin and gentamycin gave the highest activity against different salmonella isolates while amoxicillin, tetracycline, and nitrofurantoin showed the highest resistance rate. Pathogenicity of the isolated serovars was tested in chickens. All isolates were found pathogenic with various degree of virulence. SDSPAGE protein analysis for the salmonella isolated form different poultry species revealed 12 protein bands ranged from $22-289 \mathrm{kDa}$. The differences were insufficient for reliable differentiation between the isolates and accordingly, it could be used beside other molecular techniques in differentiation between the salmonella strains.
\end{abstract}

Salmonellae are widespread in the environment worldwide resulting in human and animal diseases and costing many billions of pounds each year (Morales and Thuman, 1993). Among all animal species salmonella are most frequently reported from poultry and poultry products (Gupta and Verma, 1993; Rahman et al., 1997; Abd EL-Hamid et al., 2004 and Murugkar et al., 2005). Poultry products are known to be significant reservoir for salmonella and the most important source of Salmonella enteritidis infection in humans. On the other hand, with great expansion of the poultry industry, the wide spread occurrence of avian salmonellosis has ranked it as one of the most important egg-borne bacterial diseases of poultry (Hayes et al., 1999; Davis and Berslin, 2001; Molbak and Neimann, 2002). Because of the large population at risk there is an increase active nationwide programmes for their isolation and identification (Waltman and Malison, 1995; Waltman; 2000; Rybott et al., 2004)

In recent years, antibiotic resistance in salmonellae has assumed alarming proportions worldwide (Cruchaga et al., 2001; Murughar et al., 2005).

Monitoring drug resistant pattern among salmonella isolates is vital to the clinician in regard to treatment of the diseased cases and to produce an important tool for devising a comprehensive chemoprophylactic and chemotherapeutic drug, schedule on the flock basis within geographical areas. The food and drug administration centers for disease control and prevention and others believe that agricultural misuse of antibiotics accounts for the majority of increases in antibiotic resistant isolates (Tollefson et al., 1999) and that many lead to public health threat (Witte, 1998).

Analysis of the whole-cell protein patterns has been used extensively to the study of the differences among bacterial genera, species and strains (Walia et al., 1988). Outer membrane protein analysis has proved to be useful technique in the characterization of Salmonella (Fadl et al., 2002; Ochea-Reparaz et al., 2004).

* Corresponding author. Tel.: +20 223901930

E-mail address: kamalkhalil1@yahoo.com 
The goal of this study was to elucidate the following points; i) Incidence of salmonellae in different poultry species with spotlighting the most prevalent isolated Salmonella serovars; ii) Determination of the antibiogram of different salmonella isolates; iii) Detection of the pathogenicity of different isolated serovars in day-old chicks; iv) Studying the whole-cell protein analysis of these isolates by SDS-PAGE.

\section{Materials and Methods}

Samples. A total of 620 eggs (including 450 chicken eggs, 150 duck eggs and 20 Ostrich eggs) either fertile or infertile were obtained from different farms from January 2004 to December 2005. A total of 1615 samples collected from liver, spleen, intestine ( ceacum and cecal tonsils ), bone marrow, yolk sac and heart blood of living or freshly dead cases of different poultry species ( 820 chickens, 230 ducks, 225 pigeons, 175 quails, 130 turkeys and 35 Ostriches ). The collected samples were subjected to bacteriological examination.

Isolation of Salmonellae. The isolation of Salmonellae from different samples was carried out according to (Waltman et al., 1998)

Identification of the isolates. Serological identification was done using Kauffman white scheme described by Edwards and Ewing, (1986) and Kauffmann, (1974) by using polyvalent and monovalent $(\mathrm{O})$ and $(\mathrm{H})$ antisera.

Antibiotic sensitivity test. In vitro susceptibility testing of different salmonella isolates was determined against different commercial antibiotic discs using disc diffusion method described by (Quinn et al., 1994).

Pathogenicity test. One hundred and fourty one-day-old chicks were used in this test. They were divided into 14 groups (10 birds/group). The first 13 groups were used for pathogenicity testing of different salmonella isolates, while the last one was used as non-infected control group. The test was done according to (Bakshi et al., 2003). Postmortem examination and reisolation of the infected microorganisms from internal organs were also, carried out.

Sodium Dodecyl sulphate - Polyacrylamide Gel Electrophoresis (SDS-PAGE). Total protein profile of the salmonella isolates was carried out using sodium dodecyle sulphate polyacrylamide gel electrophoresis (SDSPAGE) by the method described by Laemmli (1970) using miniprotein electrophoresis system (BioRad).The samples were electrophoresed for $1 \mathrm{~h}$ at $100 \mathrm{~V}$. Each run included prestaine protein marker (Invitrogen Co.). The gels were stained with Commassie brilliant blue and destained using distaining solution according to (Bushuk et al., 1999; Demiralp et al., 2000).

Total protein analysis. The protein analysis of the SDS-PAGE for the salmonella isolates was done using the computer analysis program (GelPro Analyzer V 3.1).

\section{Results and Discussion}

Bacteriological examination of the egg samples from different poultry species revealed an overall incidence of $1.9 \%$ of the examined samples [620], (Table 1). These results are greatly in agree with those obtained by Moustafa (1982) who recorded an incidence of $1.7 \%$ of salmonella isolation from infertile egg samples. The lowest incidence of isolation was obtained with chicken egg samples (1.6\%) that agree with the results obtained by EL-Agroudi and Awad (1966) who recorded only 88 salmonella positive eggs out of 5000 examined hen eggs which constituted an incidence of $1.7 \%$.On the other hand, incidence of salmonella isolation from duck eggs was the highest (3.3\%) and mostly identified as S. typhimurium (Table 2, 3). It was reported that in spite of the very thick cuticle covering the duck eggs, but it was observed that it may be removed as a result of being scraped by the parent or another duck while the cuticle is still soft after oviposition or due to the habit of the ducks to defecate in the nesting boxes that contaminate egg shell and increase the probability of the infection. There was an association between consumption of duck eggs and gastrointestinal illness which was largely proved to be due to the presence Salmonella typhimurium (Henry, 2000).

It was found that the most prevalent salmonella serovars isolated from eggs of different poultry species was $S$. enteritidis [41.7 5\%] (Table 2,3). Cases of salmonellosis caused by $S$. enteritidis had dramatically increased from $5 \%$ to 26\% during the period 1976 to 1994. (U.S. Department of Agriculture 1995) and the major source of these infections was properly grade A table eggs (Shah et. al. 1991). Many studies have established that $S$. enteritidis contaminates eggs when the organism is passed from the infected reproductive tissue of the hens rather than the shell, to the contents of contaminated eggs (Shivaprasad et al. 1990; Humphrey, 1994).

On the other hand, it was found that the highest incidence of salmonella isolation was obtained from ducks [7.8\%] (Table 4) which were mostly belonged to $S$. enteritidis (6 
Table (1): Incidence of salmonella isolation from egg samples from different poultry species.

\begin{tabular}{lccc}
\hline \multicolumn{1}{c}{ Examined eggs } & \multicolumn{2}{c}{ Salmonella positive eggs } \\
\hline \multicolumn{1}{c}{ Species } & No. & No. & Percent \\
\hline Chicken eggs & 450 & 7 & 1.6 \\
Duck eggs & 120 & 4 & 3.3 \\
Ostriches eggs & 50 & 1 & 2.0 \\
\multicolumn{1}{c}{ Total } & 620 & 12 & 1.9 \\
\hline
\end{tabular}

Table (2): Salmonella serovars isolated from eggs from different poultry species.

\begin{tabular}{|c|c|c|c|c|c|}
\hline \multirow{2}{*}{$\begin{array}{c}\text { Salmonella } \\
\text { serovars }\end{array}$} & \multirow{2}{*}{$\begin{array}{c}\text { Chicken } \\
\text { eggs }\end{array}$} & \multirow[t]{2}{*}{ Duck eggs } & \multirow{2}{*}{$\begin{array}{c}\text { Ostriches } \\
\text { eggs }\end{array}$} & \multicolumn{2}{|c|}{ Total } \\
\hline & & & & No. & Percent \\
\hline S. enteritidis & 3 & 1 & 1 & 5 & 41.7 \\
\hline S. typhimurium & 1 & 2 & 0 & 3 & 25.0 \\
\hline S. infantis & 2 & 0 & 0 & 2 & 16.7 \\
\hline S. montivideo & 1 & 0 & 0 & 1 & 8.3 \\
\hline S. virginia & 0 & 1 & 0 & 1 & 8.3 \\
\hline Total & 7 & 4 & 1 & 12 & 100 \\
\hline
\end{tabular}

isolates) followed by $S$. typhimurium (5 isolates). These results agreed with Hui and Das (2001) who isolated 15 salmonella strains (5.36 $\%$ ) out of 280 samples from dead, diseased and apparently healthy ducks at different farms in Bengal, India and serotyping of the isolated strains revealed that they were grouped into 2 different serogroups S. enteritidis and S. typhimurium constituting $80 \quad \%$ and $20 \%$ respectively.

Other salmonella serovars isolated from ducks includes $S$. infantis (3 isolates). $S$. montivideo (2 isolates), $S$. agona and $S$. virginia (one isolate from each). The same serovars were isolated from ducks by other authors (Simko, 1988; Maff, 1997).

The incidence of salmonella isolation from chicken samples was $4.6 \%$ (Table 4 ), including S. enteritidis $(43.2 \%)$, S. typhimurium $(18.4 \%)$, S. infantis (15.8\%), S. montivideo (10.5\%), S. cerro (7.9 \%), S. rubislaw (5.3\%), S.derby, S. poona and $S$. Kentucky (2.6\% for each) (Table $5)$.

These results are in agreement with that obtained by Hassan et al. (2003) who isolated 35 salmonella isolates $(5.51 \%)$ out of 635 examined chicken samples at different growth stages and were identified serologically to $S$. typhimurium, $S$. enteritidis, $S$. pullorum and $S$. rubislaw. Abd-EL-Hamid et al. (2004) isolated S. enteritidis, S. typhimurium, S. kentucky, S. montivideo from duck and chicken samples of different growth stages, while Murugkar et al.
(2005) isolated 231 cloacal swabs from diarrhoeic birds and the isolates were serotyped S. typhimurium (12 isolates), S. gallinarum (12 isolates), $S$. enteritidis (8 isolates) and $S$. pratyphi $\mathrm{B}$ ( 2 isolates). These results disagree with Mojnaric et al. (2003) who detected lower incidence of salmonella isolation $(2.75 \%)$ from chicken samples during the year 2002 in northwestern Croatia, mostly confirmed to be $S$. enteritidis (82\%) and other salmonellae (18\%).

Turkeys isolates constituted $7.4 \%$ of the total salmonella isolates from different poultry species (Table 5). The isolated strains were serotyped as $S$. enteritidis, $S$. typhimurium, $S$. rubislaw, $S$. derby and $S$. sandiago. The same serotypes were isolated previously by several authors (Pomeroy et al., 1984; Hirschman and Seidel, 1992; Hafez et al., 1997). Salmonellosis in turkeys is distributed world wide and resulted in severe economic losses which caused by high poult mortality during the first 4 weeks of age, high medication costs, reduction in egg production in breeder flocks, poor poult quality and high costs for eradication and control measures.

Pigeons and quails had the least incidence of salmonella isolation among different species of poultry, $1.3 \%$ and $1.2 \%$ respectively (Table 4 ). $S$. typhimurium was the most prevalent isolated serovars from quails (2 isolates), followed by $S$. agona (1 isolate) (Table 5), while the isolates from pigeons belonged to $S$. typhimurium and $S$. infantis. These results agree with Cizek et al. 
Table (3): Antigenic structure of different salmonellae isolated from poultry.

\begin{tabular}{|c|c|c|c|c|}
\hline \multirow{3}{*}{ Salmonella serovars } & \multirow{3}{*}{ Sero-group } & \multicolumn{3}{|c|}{ Antigenic structure } \\
\hline & & \multirow{2}{*}{ [O] } & \multicolumn{2}{|c|}{$[\mathrm{H}]$} \\
\hline & & & Phase (1) & Phase (2) \\
\hline S. agona & B & $1,4,12$ & $f, g, s$ & \\
\hline S. derby & B & $1,4,[5], 12$ & f,g & - \\
\hline S. sandiego & B & $4,[5], 12$ & $\mathrm{e}, \mathrm{h}$ & {$[1,2]$} \\
\hline S. typhimurium & $\mathrm{B}$ & $1,4,[5], 12$ & $\mathrm{i}$ & $\mathrm{e}, \mathrm{h}, \mathrm{Z}_{15}$ \\
\hline S. infantis & $\mathrm{C} 1$ & 6,7 & $\mathrm{r}$ & 1,2 \\
\hline S. Montevideo & $\mathrm{C} 1$ & 6,7 & $\mathrm{~g}, \mathrm{~m}, \mathrm{~s},[\mathrm{p}]$ & 1,5 \\
\hline S. kentucky & $\mathrm{C} 3$ & 8,20 & $\mathrm{i}$ & - \\
\hline S. virginia & $\mathrm{C} 3$ & 8 & $\mathrm{~d}$ & $\mathrm{Z}_{6}$ \\
\hline S. enteritidis & D1 & $1,9,12$ & $\mathrm{~g}, \mathrm{~m}$ & 1,2 \\
\hline S. rubislaw & $\mathrm{F}$ & 11 & $\mathrm{r}$ & - \\
\hline S. poona & G1 & $1,13,22$ & $\mathrm{z}$ & e,n,x \\
\hline S. cerro & $\mathrm{K}$ & $8,14,18$ & $\mathrm{Z}_{4}, \mathrm{Z}_{12}$ & 1,6 \\
\hline
\end{tabular}

Table (4): Incidence of salmonella isolation from different poultry species.

\begin{tabular}{lccc}
\hline \multirow{2}{*}{\multicolumn{1}{c}{ Poultry species }} & No. of examined cases & \multicolumn{2}{c}{ Salmonella positive cases } \\
\cline { 3 - 4 } & & No. & Percent \\
\hline Chicken & 820 & 38 & 4.6 \\
Ducks & 230 & 18 & 7.8 \\
Pigeon & 155 & 2 & 1.3 \\
Quails & 245 & 3 & 1.2 \\
Turkeys & 130 & 5 & 3.8 \\
Ostriches & 35 & 1 & 2.9 \\
\multicolumn{1}{c}{ Total } & 1615 & 67 & 4.1 \\
\hline
\end{tabular}

(1994) who isolated salmonella from carrier pigeons in salmonella-free farms as well as Hudson et al. (2000) and Mosaad et al. (2000) who isolated $S$. typhimurium from wild and domesticated quails.

Concerning isolation of salmonella from Ostrich's samples only one case was salmonella positive (Table 4) and serotyped as $S$. typhimurium. This result is in agreement with that obtained by Gopo and Banda, (1997) who reported salmonella infection from Ostrich's samples. This result also agreed with Ley et al. (2001) who detected Salmonella and Campylobacter in examined Ostrich's carcasses.

Monitoring drug resistance pattern among different isolated salmonella serovars (Table 6) revealed that the highest number of isolates showed resistance against amoxicillin (37 isolates, $46.3 \%)$ and tetracycline (34 isolates, $42.5 \%$ ) followed by nitrofurantoin (18 isolates, $22.5 \%$ ), chloramphinicol (15 isolates, $18.8 \%$ ), naldixic acid (11 isolates, 13.8\%), sulphamethoxazon-trimethoprim (9 isolates, 11.3\%) and gentamycin (7 isolates, 8.8\%). The least resist- ance rates were detected against cepheridin, norofloxacin and ciprofloxacin (4 isolates for each, $5.0 \%$ ). These results agree with HUI and Das, (2001) who found that most of salmonellae isolated from ducks were highly resistant to oxytetracycline followed by tetracycline and penicillin G. They also agree with Murugkar et al. (2005) who found that the most salmonella isolates were resistant to doxycycline $(61.05 \%)$, ampicillin (51.57\%), amoxicillin (45.26\%), tetracycline $(44.21 \%)$, nitrofurantoin $(15.79 \%)$, trimethoprim (9.5\%) and gentamycin (6.3\%). They added that flouroquinolone group of antibiotics have the least rate among salmonella isolates.

Resistance to different antibiotics is of great concern since most of these antibiotics are added in the poultry feed as supplements and the obvious lack of control on the antibiotics usage may be the probable cause for their high resistance (Dorn et al., 1992).

The pathogenicity testing of the isolated serovars in day-old chicks (Table 7) revealed that $S$. enteritidis, S. typhimurium, S. infantis and 
Table (5): Salmonella serovars isolated from different poultry species.

\begin{tabular}{|c|c|c|c|c|c|c|c|c|c|c|c|c|c|c|}
\hline \multirow{2}{*}{$\begin{array}{c}\text { Salmonella } \\
\text { serovars }\end{array}$} & \multicolumn{2}{|c|}{ Chicken } & \multicolumn{2}{|c|}{ Ducks } & \multicolumn{2}{|c|}{ Pigeon } & \multicolumn{2}{|c|}{ Quails } & \multicolumn{2}{|c|}{ Turkeys } & \multicolumn{2}{|c|}{ Ostriches } & \multicolumn{2}{|c|}{ Total } \\
\hline & No & $\%$ & No & $\%$ & No & $\%$ & No & $\%$ & No & $\%$ & No & $\%$ & No & $\%$ \\
\hline S. enteritidis & 13 & 34.2 & 6 & 33.3 & 0 & $\cdot$ & 0 & $\cdot$ & 1 & 20 & 0 & 0 & 20 & 29.9 \\
\hline S. typhimurium & 7 & 18.4 & 5 & 27.8 & 1 & 50 & 2 & 66.6 & 1 & 20 & 1 & 1.5 & 17 & 25.4 \\
\hline S. infantis & 6 & 15.8 & 3 & 16.7 & 1 & 50 & 0 & . & 0 & . & 0 & 0 & 10 & 14.9 \\
\hline S. montevideo & 4 & 10.5 & 2 & 11.1 & 0 & 0 & 0 & . & 0 & . & 0 & 0 & 6 & 9 \\
\hline S. rubislaw & 2 & 5.3 & 0 & 0 & 0 & 0 & 0 & . & 1 & 20 & 0 & 0 & 3 & 4.5 \\
\hline S. cerro & 3 & 7.9 & 0 & 0 & 0 & 0 & 0 & . & 0 & 0 & 0 & 0 & 3 & 4.5 \\
\hline S. derby & 1 & 2.6 & 0 & 0 & 0 & 0 & 0 & . & 1 & 20 & 0 & 0 & 2 & 3.0 \\
\hline S. poona & 1 & 2.6 & 0 & 0 & 0 & 0 & 0 & . & 0 & 0 & 0 & 0 & 2 & 3.0 \\
\hline S. sandiago & 0 & . & 0 & 0 & 0 & 0 & 0 & . & 1 & 20 & 0 & 0 & 1 & 1.5 \\
\hline S. Kentucky & 1 & 2.6 & 0 & 0 & 0 & 0 & 0 & . & 0 & 0 & 0 & 0 & 1 & 1.5 \\
\hline S. agona & 0 & . & 1 & 5.6 & 0 & 0 & 1 & 33.3 & 0 & 0 & 0 & 0 & 2 & 3.0 \\
\hline S. virginia & 0 & . & 1 & 5.6 & 0 & 0 & 0 & $\cdot$ & 0 & 0 & 0 & 0 & 1 & 1.5 \\
\hline Total & 38 & 56.7 & 18 & 26.9 & 2 & 3 & 3 & 4.5 & 5 & 7.5 & 1 & 1.5 & 67 & 100 \\
\hline
\end{tabular}

Table (6): Antibiotic Resistance pattern of salmonellae isolated from poultry

\begin{tabular}{|c|c|c|c|c|c|c|c|c|c|c|c|c|c|}
\hline \multirow{2}{*}{$\begin{array}{c}\text { Salmonella } \\
\text { Serotype }\end{array}$} & \multicolumn{3}{|c|}{ Source } & \multicolumn{10}{|c|}{ No. of salmonella isolates resistant to antibiotics } \\
\hline & Origin & No. & Total & $\mathbf{F}$ & NA & $\mathbf{C}$ & AML & GM & TE & $\mathbf{C E}$ & SXT & CIP & Nor \\
\hline S. enteritidis & $\begin{array}{l}\text { Eggs } \\
\text { poultry }\end{array}$ & $\begin{array}{c}5 \\
20\end{array}$ & 25 & 6 & 5 & 6 & 15 & 2 & 10 & 1 & 6 & 2 & 2 \\
\hline S. typhimurium & $\begin{array}{l}\text { Eggs } \\
\text { poultry }\end{array}$ & $\begin{array}{c}3 \\
17\end{array}$ & 20 & 5 & 3 & 4 & 8 & 1 & 8 & 1 & 1 & 0 & 0 \\
\hline S. infantis & $\begin{array}{l}\text { Eggs } \\
\text { poultry }\end{array}$ & $\begin{array}{c}2 \\
10\end{array}$ & 12 & 4 & 2 & 2 & 5 & 1 & 5 & 1 & 0 & 0 & 1 \\
\hline S. montevideo & $\begin{array}{l}\text { Eggs } \\
\text { poultry }\end{array}$ & $\begin{array}{l}1 \\
6\end{array}$ & 7 & 2 & 1 & 0 & 2 & 1 & 4 & 0 & 0 & 1 & 0 \\
\hline S. rubislaw & poultry & 3 & 3 & 0 & 0 & 1 & 2 & 0 & 1 & 0 & 1 & 0 & 1 \\
\hline S. cerro & poultry & 3 & 3 & 1 & 0 & 1 & 1 & 0 & 2 & 0 & 0 & 0 & 0 \\
\hline S. virginia & $\begin{array}{l}\text { Eggs } \\
\text { poultry }\end{array}$ & $\begin{array}{l}1 \\
1\end{array}$ & 2 & 0 & 0 & 0 & 0 & 0 & 1 & 0 & 0 & 1 & 0 \\
\hline S. agona & poultry & 2 & 2 & 0 & 0 & 1 & 1 & 0 & 0 & 0 & 1 & 0 & 0 \\
\hline S. poona & poultry & 2 & 2 & 0 & 0 & 0 & 1 & 1 & 1 & 0 & 0 & 0 & 0 \\
\hline S. derby & poultry & 2 & 2 & 0 & 0 & 0 & 0 & 0 & 1 & 1 & 0 & 0 & 0 \\
\hline S. sandiago & poultry & 1 & 1 & 0 & 0 & 0 & 1 & 1 & 0 & 0 & 0 & 0 & 0 \\
\hline S. kentucky & poultry & 1 & 1 & 0 & 0 & 0 & 1 & 0 & 1 & 0 & 0 & 0 & 0 \\
\hline Total & - & 80 & 80 & 18 & 11 & 15 & 37 & 7 & 34 & 4 & 9 & 4 & 4 \\
\hline
\end{tabular}

$\mathbf{F}=$ Nitrofurantoin $\quad \mathbf{A M L}=$ Amoxicillin

NA $=$ Naldixic acid

$\mathbf{C}=$ Chloramphinicol

$\mathbf{G M}=$ Gentamycin

$\mathbf{T E}=$ Tetracycline
SXT=sulphamethoxazone-trimethoprim

S. derby were highly pathogenic $(100 \%$ mortality) followed by, S. cerro, S. agona and $S$. virginia (95\% mortality) while $S$. rubislaw, $S$. kentucky, S. montivideo, $S$. santiago and $S$. poona were less pathogenic $(90 \%, 85 \%, 80 \%$, $80 \%$, and $80 \%$ respectively). Most of the mortalities occur within $24-48 \mathrm{~h}$ post-infection accompanied with reisolation of the injected serovars from different internal organs. The dead

$$
\begin{aligned}
& \mathbf{C E}=\text { Cephridin } \\
& \mathbf{C I P}=\text { Ciprofloxacin } \\
& \text { Nor }=\text { Norfloxacin }
\end{aligned}
$$

birds showed signs of septicemia include typical congestion of blood vessels and internal organs with mottling and enlargement of the liver and spleen. A similar picture was recorded by Synoeynbos et al. (1986) and Lee, (1987).

The variation in the degree of virulence could be attributed to the type of serovars and also the routes of infection as the chicks are more susceptible to salmonella infection by inhalation 
Table (7): Pathogenicity of salmonella serovars isolated from poultry

\begin{tabular}{|c|c|c|c|c|c|c|c|c|c|c|}
\hline \multirow{2}{*}{$\begin{array}{c}\text { Salmonella } \\
\text { Serovars }\end{array}$} & \multirow{2}{*}{$\begin{array}{l}\text { No. of } \\
\text { infected } \\
\text { chicks }\end{array}$} & \multirow{2}{*}{$\begin{array}{c}\text { Route } \\
\text { of } \\
\text { infection }\end{array}$} & \multirow{2}{*}{$\begin{array}{c}\text { Dose of } \\
\text { infection } \\
\text { CFU }\end{array}$} & \multicolumn{2}{|c|}{ Mortality } & \multicolumn{5}{|c|}{ Salmonella re-isolation } \\
\hline & & & & No. & $\%$ & Heart & Liver & $\begin{array}{c}\text { Bone } \\
\text { marrow }\end{array}$ & Lungs & kidney \\
\hline Control -ve & 20 & & 0 & 0 & 0 & 0 & 0 & 0 & 0 & 0 \\
\hline S. enteritidis & 20 & & & 20 & 100 & $20 / 20$ & $20 / 20$ & $15 / 20$ & $18 / 20$ & $16 / 20$ \\
\hline S. typhimurium & 20 & & & 20 & 100 & $20 / 20$ & $20 / 20$ & $18 / 20$ & $15 / 20$ & $16 / 20$ \\
\hline S. infantis & 20 & & & 20 & 100 & $20 / 20$ & $20 / 20$ & $18 / 20$ & $19 / 20$ & $15 / 20$ \\
\hline S. derby & 20 & & & 20 & 100 & $20 / 20$ & $20 / 20$ & $18 / 20$ & $19 / 20$ & $15 / 20$ \\
\hline S. cerro & 20 & & 空 & 19 & 95 & $18 / 19$ & $19 / 19$ & $17 / 19$ & $15 / 19$ & $13 / 19$ \\
\hline S. virginia & 20 & a & & 19 & 95 & $19 / 19$ & $19 / 19$ & $17 / 19$ & $19 / 19$ & $17 / 19$ \\
\hline S. agona & 20 & こ & $\infty$ & 19 & 95 & $18 / 19$ & $19 / 19$ & $16 / 19$ & $17 / 19$ & $15 / 19$ \\
\hline S. rubislaw & 20 & & $\bar{x}$ & 18 & 90 & $18 / 18$ & $18 / 18$ & $16 / 18$ & $15 / 18$ & $14 / 18$ \\
\hline S. kentucky & 20 & & & 17 & 85 & $14 / 17$ & $17 / 17$ & $14 / 17$ & $16 / 17$ & $15 / 17$ \\
\hline S. montevideo & 20 & & & 16 & 80 & $16 / 16$ & $16 / 16$ & $13 / 16$ & $15 / 16$ & $10 / 16$ \\
\hline S. poona & 20 & & & 16 & 80 & $13 / 16$ & $16 / 16$ & $11 / 16$ & $12 / 16$ & $11 / 16$ \\
\hline S. sandiago & 20 & & & 16 & 80 & $15 / 16$ & $16 / 16$ & $10 / 16$ & $14 / 16$ & $13 / 16$ \\
\hline
\end{tabular}

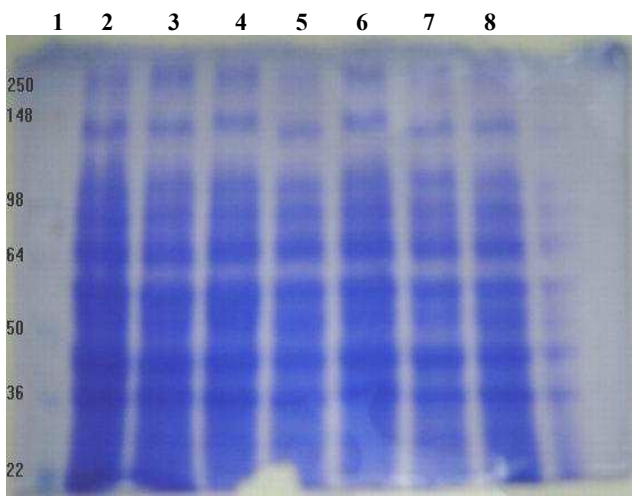

Fig (1): SDS-PAGE protein profile of salmonella isolated from different species of poultry and eggs. Lane (1): protein marker, Lane (2):S.rubislaw, Lane (3):S.sandiago, Lane (4):S.derby, Lane (5):S.typhimurium, Lane (6):S.agona, Lane (7):S.virginia, Lane (8):S.enteritidis.

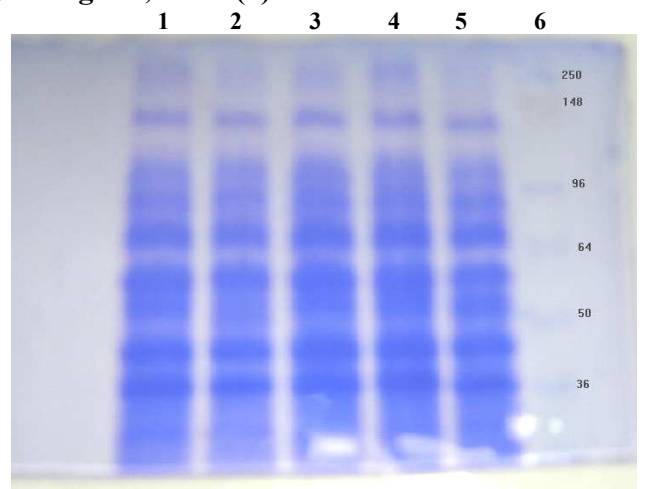

Fig (2): SDS-PAGE protein profile of salmonella isolated from chicken and chicken eggs. Lane (1): S.montevideo, Lane (2):S.kentucky, Lane (3):S.infantis, Lane (4):S.cerro, Lane (5):S.poona, Lane (6): protein marker. and parentral routes than via oral route (Baskerville et al., 1992; Poppe et al., 1993; Cooper et al., 1994).

SDS-PAGE protein analysis of the isolated salmonella from different poultry revealed about 12 protein bands (Tables 8,9, Fig 1,2 ). The protein bands ranged from $22-289 \mathrm{kDa}$. The differences were insufficient for reliable differentiation between the isolates. The $29 \mathrm{kDa}$ protein band was the common antigen which represent SCOPA as mentioned by Soad,(1995), while there were protein bands $67,57,43,36$, $33,22 \mathrm{kDa}$ in all the isolates which agree with the results of Ochea-Reparaz et al. (2004) who mentioned that Flagellin 53 and $45.1 \mathrm{kDa}$, Porins 35-36 kDa Omp A $34 \mathrm{kDa}$ and Omp 22.1 $\mathrm{kDa}$, Nese et al. (2003) who found that the $S$. typhimurium isolates contained OMPs with the molecular sizes $70 \mathrm{kDa}$ and the highest antigenicity common protein fraction was 36-43 kDa. and Helmuth et al. (1985) who mentioned that the $S$. typhimurium strains generally contained OMPs of 37, 40 and $41.7 \mathrm{kDa}$. The protein band of 119.6 was only in S. virginia and S. typhimurium.

In our results, the differences were insufficient for reliable differentiation and the protein analysis may be used beside other molecular techniques to differentiate among the salmonella strains. 
Table (8): SDS-PAGE whole cell protein analysis of salmonella isolated from different species of poultry and eggs.

\begin{tabular}{cccccccc}
\hline $\begin{array}{c}\text { Protein } \\
\text { Marker }\end{array}$ & $\begin{array}{c}\text { Lane 2 } \\
\text { S.rubislaw } \\
\text { (Turkey) }\end{array}$ & $\begin{array}{c}\text { Lane 3 } \\
\text { S.sandiago } \\
\text { (Turkey) }\end{array}$ & $\begin{array}{c}\text { Lane 4 } \\
\text { S.derby } \\
\text { (Turkey) }\end{array}$ & $\begin{array}{c}\text { Lane 5 } \\
\text { S.typhimurium } \\
\text { (Ostrich) }\end{array}$ & $\begin{array}{c}\text { Lane 6 } \\
\text { S.agona } \\
\text { (Quail) }\end{array}$ & $\begin{array}{c}\text { Lane 7 } \\
\text { S.virginia } \\
\text { (Duck's } \\
\text { egg) }\end{array}$ & $\begin{array}{c}\text { Lane 8 } \\
\text { S.enteritidis } \\
\text { (Duck's egg) }\end{array}$ \\
\hline \multirow{2}{*}{250} & 289.1 & 289.1 & 289.1 & 289.1 & 289.1 & 289.1 & 289.1 \\
148 & & & & & & & \\
& 140.3 & 140.3 & 140.3 & & 140.3 & & 140.3 \\
& 105.4 & 105.4 & 105.4 & 105.4 & 105.4 & 105.4 & 105.4 \\
98 & & & & & & & \\
& 87.8 & 87.8 & 87.8 & 87.8 & 87.8 & 87.8 & 87.8 \\
64 & 67.3 & 67.3 & 67.3 & 67.3 & 67.3 & 67.3 & 67.3 \\
& 57.9 & 57.9 & 57.9 & 57.9 & 57.9 & 57.9 & 57.9 \\
50 & 51.9 & 51.9 & 51.9 & 51.9 & 51.9 & 51.9 & 51.9 \\
& 43.3 & 43.3 & 43.3 & 43.3 & 43.3 & 43.3 & 43.3 \\
& 36.4 & 36.4 & 36.4 & 36.4 & 36.4 & 36.4 & 36.4 \\
36 & 29.1 & 29.1 & 29.1 & 29.1 & 29.1 & 29.1 & 29.1 \\
& 24.1 & 24.1 & 24.1 & 24.1 & 24.1 & 24.1 & 24.1 \\
& 22 & 22 & 22 & 22 & 22 & 22 & 22 \\
\hline
\end{tabular}

Table (9): SDS-PAGE whole cell protein analysis of salmonella isolated from chicken and chicken eggs.

\begin{tabular}{cccccc}
\hline $\begin{array}{c}\text { Lane 1 } \\
\text { S.montevideo } \\
\text { (chicken eggs }\end{array}$ & $\begin{array}{c}\text { Lane 2 } \\
\text { S.kentucky } \\
\text { (chicken) }\end{array}$ & $\begin{array}{c}\text { Lane 3 } \\
\text { S.infantis } \\
\text { (chicken) }\end{array}$ & $\begin{array}{c}\text { Lane 4 } \\
\text { S.cerro } \\
\text { (chicken) }\end{array}$ & $\begin{array}{c}\text { Lane 5 } \\
\text { S.poona } \\
\text { (chicken) }\end{array}$ & $\begin{array}{c}\text { Protein } \\
\text { Marker }\end{array}$ \\
\hline 289.1 & 289.1 & 289.1 & 289.1 & 289.1 & 250 \\
& & & & & 148 \\
134.1 & 134.1 & 134.1 & 134.1 & 134.1 & 98 \\
& & & & & 67.3 \\
67.1 & 67.3 & 67.3 & 67.3 & 67.3 & 64 \\
57.9 & 57.9 & 57.9 & 57.9 & 57.9 & \\
52.4 & 52.4 & 52.4 & 52.4 & 52.4 & \\
& & & & & 50 \\
44.9 & 44.9 & 44.9 & 44.9 & 44.9 & 36 \\
36.2 & 36.2 & 36.2 & 36.2 & 36.2 & \\
23 & 33 & 33 & 33 & 33 & \\
24.1 & 29 & 29 & 29 & 29 & \\
22 & 24.1 & 24.1 & 24.1 & 24.1 & \\
\hline
\end{tabular}




\section{References}

Abd El-Hamid, H. S.; Torkey, H. A.; AL Shaboury, F. A.; Sleim, M. M. A.; Ellakany and Awad, A. M. (2004): Epidemiological studies on Salmonellosis in poultry. The $4^{\text {th }}$ Sci. Conf., 2-4 $4^{\text {th }}$ Oct. 2004, Fac. Med. Alex. Univ., Egypt.

Bakshi, C. S.; Singh, V. P.; Malik, M.; Singh, R. K. and Sharma, B. (2003): $55 \mathrm{~kb}$ plasmid and virulence associated genes are positively correlated with Salmonella enteritidis pathogenicity in mice and chickens. Vet. Res. Commu., 2: 425-432.

Baskerville, A.; Humphery, T. J.; Fitzgeorge, R. B.; Cook, R.W.; Chart, H.; Rowe, B. and Whitehead, A. (1992): Airborne infection of laying hens with S.enteritidis phage type 4. Vet. Rec., 130: 395-398.

Bushuk, W.; Hay, R. L.; Larsen, N. G.; Sara, R. G.; Simmons, L. D. and Sutton, K. H. (1999): Effect of mechanical dough development on the extraclability of wheat storage proteins from bread dough. Cereal Chem.74: 389-395.

Cizek, A.; Literak, I.; Heijlicek, K.; Treml, F. and Smola, J. (1994): Salmonella contamination of the environment and its incidence in wild birds. J. Vet. Med., 41:320-329.

Cooper, G. I.; Venables, L. M.; Woodward, M. J. and Hormaeche, C. E. (1994): Invasiveness and persistence of Salmonella enteritidis, Salmonella typhimurium and a genetically defined $S$. enteritidis oro A strain in young chicks. Infec. Immun., 62: 4739-4746.

Cruchaga, S.; Echeita, A.; Aladwena, A.; Garcia-Pena, J.; Frias, N. and Usera, M. A. (2001): Antimicrobial resistance in Salmonellae from humans, food and animals in Spain in 1998. J. Antimicrob. Chemoth.. 47: 315-321.

Davies, R. H. and Breslin, M. (2001): Enviromental contamination and detection of Salmonella enterica serovar Enteritidis in laying floks. Vet. Rec., 149: 699-704.

Demiralp, H.; Celik, S. and Koksel, H (2000): Effect of oxidizing agents and defatting on the electrophoretic patterns of flour proteins during dough mixing. Eur. Food Res. Tech., 211: 322-325.

Dorn, C. R.; Silapanuntakul, R.; Angrick, E. J. and Shipman, L. D. (1992): Plasmid analysis and epidemiology of salmonella enteritidis infection in three commercial layer flocks. Avian Dis., 36: 844-851.

Edwards, P. R. and Ewing, W. H. (1986): Identification of Enterobacteriaceae. $4^{\text {th }}$ ed., Minnesota, Burgess Pub. Co. EL-Agroudi, M. A. and Awad, F. I. (1966): Salmonella in hen eggs. J.Vet.Sci., (U.A.R.),3 (1): 1-5.

Fadl, A. A.; Venkilanarayanan, K. S. and Khan, M. I. (2002): Identification of Salmonella enteritidis outer membrane proteins expressed during attachment to human intestinal epithelial cells. J. Applied Microbiol., 92 (1): 180-186.

Gopo, J. M. and Banda, G. N. (1997): Occurrence of salmonella on meat and products in an Ostrich abattoir as determined with a DNA probe. South Afr. J. Animal Sci. 27 (1): 1-6.

Gupta, B. R. and Verma, J. L. (1993): Monograf on animal Salmonellosis. Izatnagar: National Salmonella Center (Veterinary), Division of Bacteriology and Mycology, IVRI, pp 9-11.

Hafez, H. M.; Stadler, A. and Kosters, J.(1997): Surveillance on salmonella in Turkey floks and processing plants. Deutsche Tieraerztliche Wochenschrift, 104: 33-35.

Hassan W. M. M.; Oraby, F. A. I. and Hassan, A. M. (2003): Comparative studies on different isolates of avian salmonellae by sodium dodocyl sulphate polyacrylamide gel electrophoresis (SDS-PAGE). J. Egypt. Med. Assoc.63 (2): $65-72$.

Hayes, S.; Nylen, G.; Smith, R.; Salmon, R. L. and Palmer, S. R. (1999): Undercooked hens eggs remain a risk factor for sporadic Salmonella enteritidis infection. Commun. Disease Pub. Hlth., 2: 66-67.

Helmuth, R.; Stephan, R.; Bunge, C.; Hoog, B.; Steinbeck, A. and Bulling, E. (1985): Epidemiology of virulence associated plasmids and outer membrane protein patterns within seven common salmonella serotypes. Infect. Immun., 48 (1): 175-182.

Henry, R. R. (2000): Salmonella infection in ducks. In: salmonella in Domestic animals. ed. By Wray. C. and Wray, A., Cabi Pub., CAB international, Wallingford. UK. pp. $157-168$.

Hirshmann, R. U. and Seidel, A.(1992): Die Entwicklung des Salmonella-Geschehens bei der Tierart Rind in Mecklenburg, Vorpommern von 1980 bis 1989. Tieraeztliche Umschau, 47: 249- 257.

Holt, J. G.; Krieg, N. R.; Sneath, P. H. A.; Staley, J. T. and William, S. T. (1996): Bergy's Manual of Determinative Bacteriology. $9^{\text {th }}$ ed., Williams and Wilkings, Baltmore, Maryland, U.S.A.

Hudson, C. R.; Quist, C.; Lee, M. D.; Keyes, K.; Dodson, S. V.; Morales, C.; Sanches, S.; White, D. G. and Maurer, J. J. (2000): The genetic relatedness of salmonella from non-domestic birds in southern United States. J.Clin.Microbiol., 38: 1860-1865.

Hui, A. K. and Das, R. (2001): Studies on isolation, serotyping and antibiotic sensitivity of salmonella isolated from ducks. Ind. Vet. J., 78 (11): 1058-1059.

Hunphrey, T. J. (1994): Contamination of egg shell and contents with Salmonella enteritis: a review. Int. J. Food Microbiol., 21: 31-40.

Kauffmann, F. (1974): Kaufman White Scheme, WHO. B.O.172, Rev.1, Acta Path. Microbiol. Scand. 61,385.

Laemmli, U. K. (1970): Cleavage of structural proteins during the assembly of the head of bacteriophage T4. Nature, 224:680-684.

Lee, G. M. (1987): Infection and immune responses to S.typhimurium in chickens. Dissert. Abstracts Inter., 47 (5): 1857.

Ley, E. C.; Morishita, T. Y.; Brisker, T. and Harr, B. S. (2001): Prevalence of Salmonella, Campylobacter and Escherichia coli on Ostrich carcasses and susceptibility of Ostrich-origin E. coli isolates to various antibiotics. Avian Dis., 45 (3): 696-700.

MAFF (1997): Salmonella in livestock production. Vet. Lab. Agency, New Haw, Addlestone.

Mojnaric, D.; Bacanek, B.; Sukalic, T. and Pavaljok, I (2003): Frequency of Salmonella infections in the poultry in the north-western Croatia. In Simpazij Perodarski Dani 2003. Edit. by Wittner, V., 164-166 ISBN 953-96259-5-5. Zegrgb, Croatian Vet. Inst. Poultry Center.

Molbak, K. and Neimann, J. (2002): Risk factors for sporadic infection with Salmonella enteritidis, Denmark, 1997-1999. Am. J. Epidemiol., 156: 654-661.

Morales, R. A. and Thurman, W. N. (1993): Methods for analyzing the effects of Salmonella outbreak on poultry prices. Prev.Vet. Med., 16: 65-66.

Mosaad, A. A.; El-Shorbagy, M. A. and El-Boraei, I. M. (2000): Studies on salmonellosis in quails. Minufiya Vet.J., 1 (1):119-129.

Moustafa, F. M. (1982): Microbiological and serological studies on avain salmonellosis. Ph.D. thesis (Microbiology), Fac. Vet. Med., Cairo Univ., Egypt. 
Murugkar, H. V.; Rahman, H.; Komar A. and Bhattacharyya, D. (2005): Isolation, phage typing and antibiogram of Salmonella from man and animals in northern India. Ind. J. Med. Res., 122:237-242.

Nese, A.; Sayhan, O.; Karacavus, A. and Torec, K. (2003) : Immunogenecity and specificity of Salmonella typhimurium outer membrane antigens. J. Cell Mol. Biol., 2: 91-97

Ochea-Reparaz, J.; Sesma, B.; Alvarez, M.; Jesus, M.; Juan, M. and Gamazo, C. (2004): Humoral immune response in hens naturally infected with salmonella enteritidis against outer membrane proteins and other surface structural antigens. Vet. Res., 35: 291-298.

Pomeroy, B. S.; Nagoraja, K. V.; Olson, H.; Ausherman, L. T.; Nivas, S. C. and Kumar, M. C. (1984): Control of salmonella infection in turkeys in Minnesota. In: proceedings of the international symposium on salmonella, New Orleans, Louisiana, pp. 115-123.

Poppe, C. ; Demczuk, W. ; McFadden, K. and Johnson, R.P. (1993): Virulence of Salmonella enteritidis phage types 4,8 and 14 and other salmonella spp. For day old chicks, hens and mice. Canad. J. Vet. Res., 57: 281-287.

Quinn, P. J.; Carter, M. E.; Markry, B. K. and Carter, G.R. (1994): Clinical Veterinary Microbiology. Wolf, London, New York, pp.237-242.

Rahman, H. ; Barman, N. N. ; Patgiri, G. P. and Kalita, N. (1997): Outbreak of Salmoellosis in broiler flocks in Assam. Indian J. Comp. Microbiol. Immunol. Infect. Dis., 18: $56-58$.

Rybolt, M. L. ; Wills, R. W. ; Byrd, J. A. ; Doler, T. P. and Bailey, R. H. (2004): Comparison of four salmonella isolation techniques in four different inoculated matrices. Poult. Sci., 83: 1112-1116.

Shah, D. B., Bradshaw, J. G. and Peeler, J. T. (1991): Thermal resistance of egg associated epidemic strains of Salmonella enteritis. J. Food Sci., 56: 391-393.

Shivaprasad, H. L.; Timoney, J. F.; Morales, S.; Lucio, B. and Baker, R. C. (1990): Pathogenesis of salmonella enteritis infection in laying chickens. I- Studies on egg transmission, clinical signs, fecal shedding and serologic responses. Avian Dis., 34: 548-557.

Simko, S. (1988): Salmonellae in ducks and geese on farms with latent infections and in centers of salmonellosis. Immunoprophylaxia (1-2): 92-101.

Soad, A. A. N. (1995): Refinement of a common antigen for detection of fowl paratyphoid carriers . PhD. Thesis, Cairo Univ., Fac. Vet. Med., Dept. Poult. Dis., Egypt.

Synoenbos, G. H.; Weinack, O. H.; Miller, B. M.; Woodward, E. D. and Weston, C. R. (1986): Large Scale trials to study competitive exclusion of salmonella in chickens . Avian Dis., 29 (4): 1004-1011.

Tollefson, L.; Fedorka-Cary, P. J. and Angulo, F. J. (1999): Public health aspects of antibiotic resistance monitoring in the USA. Acta Vet. Scand. Suppl., 92: 67-75. U.S. Department of Agriculture (1995): Tracking Food born pathogens from farm to table: data needs to evaluate control options . Conference proceedings, 9-10 January 1995. Washington, DC.

Walia, T. M.; Williamson, T.; Kaiser, A. and Tewari, R. (1988): Usefulness of protein patterns, serotyping and plasmid DNA profiles in the epidemiologic finger printing of $P$. aeruginosa. Eur. J. Microbiol. Infact. Dis., 7: 248-255. Waltman, W. D. (2000): Methods for the cultural isolation of salmonella. In: salmonella in Domestic animals, Edit by Wray, and Wray, A. CABI publishing, CAB international, Wallingford, Oxon. Ox 10 \& DE, U.K.

Waltman, W. D. ; and Mallinson, E. T.(1995): Isolation of Salmonella from poultry tissue and environmental samples: a nationwide survey. Avian Dis., 39: 45-54.

Waltman, W. D. ; Gast, R. K. and Mallinson, E. T. (1998): Salmonellosis. In: A laboratory manual for the isolation and identification of avian pathogens. $4^{\text {th }}$ ed., Swayne, D.E.; Glisson, J.R.; Jackwood, M.W.; Pearson, J.E. and Reed, W.M., Amer. Assoc. Avian Pathol., Pennsylvania Univ. New Bolton Center, Kennett Square, PA. 19348-1692.

Witte, W. (1998): Medical consequences of antibiotic used in agriculture. Science, 279: 996-997.

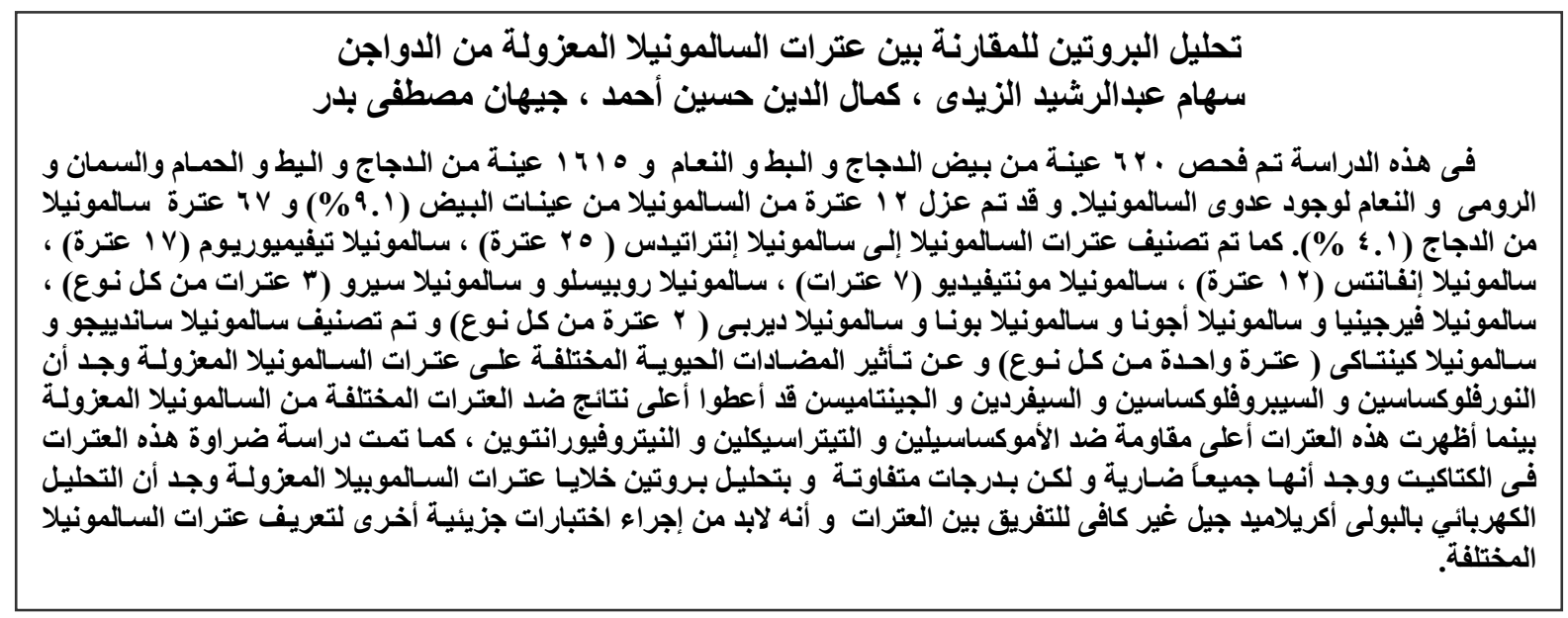

